\title{
Athanasius Handbuch
}

\author{
Hrsg. v. Peter Gemeinhardt
}

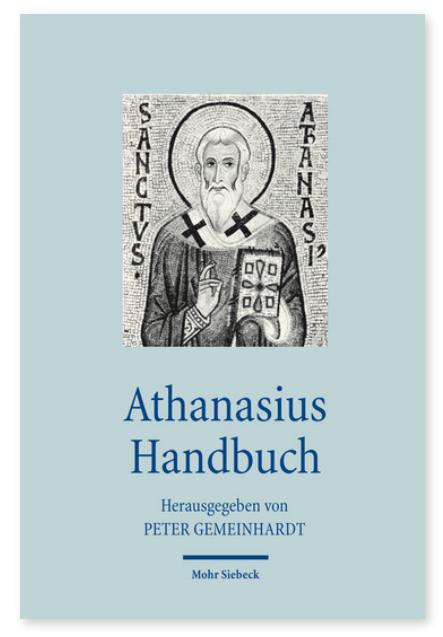

2011. XV, 578 Seiten. HdTh

ISBN 978-3-16-151653-5 DOI 10.1628/978-3-16-151653-5 eBook PDF 119,00€

ISBN 978-3-16-150078-7 fadengeheftete Broschur 29,00€ ISBN 978-3-16-150079-4 Leinen $119,00 €$
Das Athanasius Handbuch nimmt Leben, Werk und Wirkung des Bischofs Athanasius von Alexandrien (gest. 373) aus unterschiedlichen Perspektiven in den Blick: Beschrieben werden zunächst sein Umfeld und seine Zeit mit ihren theologischen, politischen, sozial- und geistesgeschichtlichen Konturen, seine Biographie und seine Beziehungen zu zeitgenössischen Personen und Gruppierungen. Sodann wird eine detaillierte Darstellung der einzelnen Schriften des Athanasius geboten, einmündend in eine systematische Rekonstruktion theologischer Leitlinien. Schließlich enthält das Handbuch einen ausführlichen Teil zu der bislang wenig erforschten Wirkungs- und Rezeptionsgeschichte des Athanasius in Kirche, Theologie und Kunst von der Antike bis zur Neuzeit. Das Handbuch macht sowohl die Persönlichkeit des alexandrinischen Bischofs als auch die Einflüsse, die ihn prägten und die von ihm selbst ausgingen, in ihrer Vielfalt und wechselseitigen Bezogenheit deutlich. Über die bisherige, meist auf einzelne Segmente seines Wirkens konzentrierte Forschung hinausgehend wird so eine Zusammenschau von Athanasius' kirchlichem, theologischem und politischem Wirken ermöglicht. Beigetragen haben dazu über dreißig Autorinnen und Autoren, die die deutschsprachige, aber auch die internationale patristische Forschung repräsentieren. Das Handbuch zieht damit eine Summe der laufenden Forschungen zu Athanasius und seiner Zeit. Es zielt darauf ab, die vorliegenden Erkenntnisse Studierenden, Lehrenden und an der Alten Kirche Interessierten in übersichtlicher Form zugänglich zu machen, zugleich aber auch neue Forschungen über die herkömmlichen Fragestellungen hinaus anzuregen.

Inhaltsübersicht

\section{A. Orientierung}

I. Textüberlieferung: Handschriften und frühe Drucke (Annette von Stockhausen)

II. Zum Stand der Athanasius-Forschung am Beginn des 21. Jahrhunderts (Hanns Christof Brennecke )

B. Person

I. Kontexte

1. Das Christentum in Alexandrien bis ins frühe 4. Jahrhundert ( Alfons Fürst)

2. Die Lage der Kirche unter Konstantin und seinen Nachfolgern (Martin Wallraff)

3. Die Kirche Ägyptens im 4. Jahrhundert (Andreas Müller)

4. Das Mönchtum in Ägypten (Barbara Müller)

5. Der »arianische« Streit (Winrich Löhr)

II. Wirkungsfelder

Vorbemerkung: Athanasius zwischen Biographie und Hagiographie ( Peter Gemeinhardt)

1. Herkunft, Jugend und Bildung (Peter Gemeinhardt)

2. Der Bischof von Alexandrien (Tobias Georges)

3. Theologie und Kirchenpolitik (Peter Gemeinhardt)

4. Askese und Spiritualität (Andreas Müller)

III. Beziehungen

1. Athanasius und Alexandrien (Winrich Löhr)

2. Athanasius und die Melitianer (Andreas Müller)

3. Athanasius und die Eusebianer (Markus Vinzent)

4. Athanasius und Markell von Ankyra (Markus Vinzent)

5. Athanasius und der Westen (Markus Vinzent)

6. Athanasius und die Kaiser (Karen Piepenbrink)

7. Athanasius und die Homöer und Homöusianer (Winrich Löhr)

8. Athanasius und Antonius (Samuel Rubenson)

9. Athanasius und Serapion von Thmuis (Klaus Fitschen)

10. Athanasius und Antiochien (Johannes Zachhuber)

11 Athanasius und Apollinaris (Silke-Petra Bergjan)

12. Athanasius und Basilius (Volker Henning Drecol/)

C. Werk

I. Schriften

1. Das apologetische Doppelwerk (Uta Heil)

2. Apologetik im Zusammenhang mit dem »arianischen Streit« (Werner Portmann, Karen Piepenbrink)

3. Schriften gegen Eusebianer und Homöer (Markus Vinzent, Uta Heil, Blossom Stefaniw)

4. Pronizänische Schriften seit 362 (Peter Gemeinhardt, Annette von Stockhausen)

5. Schriften zur Christologie (Silke-Petra Bergjan)

6. Schriften zur Pneumatologie: Epistulae ad Serapionem (Blossom Stefaniw)

7. Monastische Schriften (Dmitrij Bumazhnov)

8. Traktate zur weiblichen Askese (Dmitrij Bumazhnov)

9. Exegetische Schriften (Thomas Böhm)

10. Die Osterfestbriefe (Alberto Camplani)

II. Themen

1. Die Heilige Schrift (James D. Ernest)

2. Der dreieinige Gott (A dolf Martin Ritter)

3. Christus der Logos (Adolf Martin Ritter)

4. Der heilige Geist (Adolf Martin Ritter) 
5. Der Mensch (Stefanie Frost)

6. Erlösung (Stefanie Frost)

7. Kirche (Peter Gemeinhardt)

D. Wirkung und Rezeption

I. Rezeption und Fortschreibung in der Spätantike

1. (Pseudo-) Athanasius als Autorität im trinitarischen Streit (Markus Vinzent, Peter Gemeinhardt, Patrick Andrist)

2. (Pseudo-) Athanasius als Autorität im christologischen Streit (Benjamin Gleede)

3. Spätantike Historiographie und Hagiographie (Peter Gemeinhardt)

4. Lateinische Übersetzungen (Christian Müller)

5. Athanasius-Exzerpte in den Psalmenkatenen (Ekkehard Mühlenberg)

6. Das Symbolum Athanasianum (Volker Henning Drecoll)

II. Rezeption in den Orientalisch-Orthodoxen (nichtchalkedonensischen) Kirchen

1. Koptische Tradition (Bernd Witte)

2. Syrische Tradition (Karl Pinggéra)

3. Armenische Tradition (Anahit Avagyan)

III. Rezeption im griechischen und lateinischen Mittelalter

1. Der Osten ( Peter Gemeinhardt)

2. Der Westen (Volker Leppin)

3. Die Filioque-Kontroverse auf dem Konzil von Florenz ( Hanns Christof Brennecke )

4. Athanasius in der Ikonographie (Karin Krause)

IV. Rezeption in Reformation und Neuzeit

1. Athanasius in der Sicht der Reformatoren (Hanns Christof Brennecke)

2. Fallstudien zur neuzeitlichen Athanasius-Rezeption (Hanns Christof Brennecke, Klaus Fitschen, Ekkehard Mühlenberg)

V. Athanasius: ein ökumenischer Kirchenvater? (Peter Gemeinhardt)

Peter Gemeinhardt Geboren 1970; 1990-96 Studium der Ev. Theologie an den Universitäten Marburg und Göttingen; 200 Promotion zum Dr. theol. an der Philipps-Universität Marburg; 2003 Ordination zum Pfarrer der Evangelischen Kirche von Kurhessen-Waldeck; 2006 Habilitation an der Friedrich-Schiller-Universität Jena; seit 2007 Lehrstuhlinhaber für Kirchengeschichte an der Georg-August-Universität Göttingen; 2015-20 Sprecher des Sonderforschungsbereichs »Bildung und Religion«; 2021-23 Dekan der Theologischen Fakultät. https://orcid.org/0000-0003-3879-0397

Jetzt bestellen:

https://mohrsiebeck.com/buch/athanasius-handbuch-9783161516535?no cache=1

order@mohrsiebeck.com

Telefon: +49 (0)7071-923-17

Telefax: $+49(0) 7071-51104$ 